\title{
Cost model for evaluation of SDMB service over integrated satellite-terrestrial UMTS networks ${ }^{1}$
}

\author{
Pasquale Pace, Salvatore Marano \\ University of Calabria - Cosenza - ITALY \\ \{ppace, marano\}@deis.unical.it
}

\begin{abstract}
The number of broadband users has been growing rapidly during the last years. It is not only the number of users that increases but also the average data volume per user; a consequence of the increased number of users connected via broadband techniques is that the demand for audio and video content is also increasing.

In this paper we describe an integrated satelliteterrestrial UMTS architecture investigating on the minimization of the delivery cost. The proposed telecommunication system can offer SDMB (Satellite Digital Multimedia Broadcast) services to mobile users through the satellite or terrestrial UMTS downlink segment. On the inside of this scenario we propose a simple and efficient cost model for choosing the more suitable bearer (satellite or terrestrial) in order to save service delivery cost, moreover we design a new signalling strategy based on users location information for supporting this optimal choice.

The simulations performed show the goodness of the proposed strategy also with several mobile operator networks varying the number of users asking for the SDMB service.
\end{abstract}

Keywords-component: SDMB, 3G, UMTS, W-CDMA.

\section{INTRODUCTION}

Broadcasting technology and services have been around almost as long as the radio communication itself, and have for more than half a century, been a "broadband" channel to the user. The development now is towards fully digital broadcast networks with return channels enabling outreaching two-way communication in addition to traditional broadcast services.

Several initiatives and projects have emerged around the world to provide multimedia services using an hybrid satellite/terrestrial infrastructure to provide global coverage in a cost effective manner [1][2][3].

In the context of a growing market toward mobile multimedia, many studies to the innovative concept of Satellite Digital Multimedia Broadcast (SDMB), have been conducted [4][5][6].

The purpose of the Satellite Digital Multimedia Broadcast system is to provide capacity for several mobile operators to deliver cost effective streaming and download services directly to mobile handsets over satellite and terrestrial UMTS in both outdoor and indoor environments.

In addition, it aims at complementing $2 \mathrm{G}$ and $3 \mathrm{G}$ mobile system by achieving the true anywhere and anytime challenge with a dependable infrastructure offering point to point and point to multipoint services via satellite over zones not covered by the terrestrial networks.

\author{
Zhili Sun, Barry Evans \\ University of Surrey - Guildford - UK \\ \{Z.Sun, B.Evans\}@surrey.ac.uk
}

In this paper we propose a cost model analysis for helping the BM-SC (Broadcast Multicast - Service Center) module to carry out an efficient choice for the data transmission over the satellite or terrestrial UMTS bearer. This cost analysis strategy is greatly based on some information about the number and the location of all users that are interested in receiving the SDMB service.

The used network architecture for the proposed choice strategy consists of bi-directional channel between the UE and the BM-SC which may be supported over either GPRS or UMTS-T bearer provided by the terrestrial Mobile Network Operator. Moreover, this architecture, also involves the ability to make use of multimedia broadcast/multicast services (MBMS) [7] provided by the terrestrial Mobile Network Operator in addition to the SDMB broadcast over satellite. In this way, a second option for routing of SDMB services to the end users is provided.

This paper first presents the SDMB system architecture, then explains the cost model and decision strategies, finally concludes the paper as well as plants out future research directions.

\section{SYSTEM ARCHITECTURE OVERVIEW}

The system relies on a hybrid satellite \& terrestrial infrastructure operating in the IMT2000 core frequency band allocated to Mobile Satellite Systems.

It makes use of the UTRA FDD WCDMA waveform in full compliance with the relevant 3GPP standard to allow a very low impact on the cost of $3 \mathrm{G}$ handset. The system relies on a very high power geo-stationary satellite able to overcome indoor penetration constraints in rural areas.

An high level overview of the generic SDMB architecture is shown in fig. 1 developed in the EU F6 MAESTRO project [1].

It illustrates the key functional elements within the network that will be used to support the SDMB service. These elements include:

- SDMB-capable User Equipment; a dual mode TUMTS / S-UMTS mobile device enhanced to support SDMB services.

- SDMB Satellite that will support the transmission of SDMB services to defined coverage areas.

- SDMB Terrestrial Repeaters that may be deployed to enhance SDMB signal availability for UEs in urban areas by retransmitting the satellite signal on the ground.

- SDMB Hub that controls broadcast transmission over the SDMB satellite system taking as input media streams from the BM-SC.

- BM-SC (Broadcast Multicast - Service Center) that controls all aspects of the delivery of SDMB services including authentication and authorization of subscribers,

\footnotetext{
${ }^{1}$ This work was supported by the European Commission within the frame of the IST/FP6 integrated project MAESTRO.

The authors would like to thank all project partners for their contributions to this work.
} 
through delivery of services over the SDMB network, to service accounting.

- Content Provider that will provide the multimedia content to be delivered over the SDMB system.

The Gmb and Gi interfaces towards the SDMB Hub only need to be able to support the Broadcast Mode defined in [7], furthermore those interfaces may need to support functionality / attributes which are specific to the SDMB system. For these reasons we refer to the interfaces as Gmb* and $\mathrm{Gi}^{*}$ respectively.

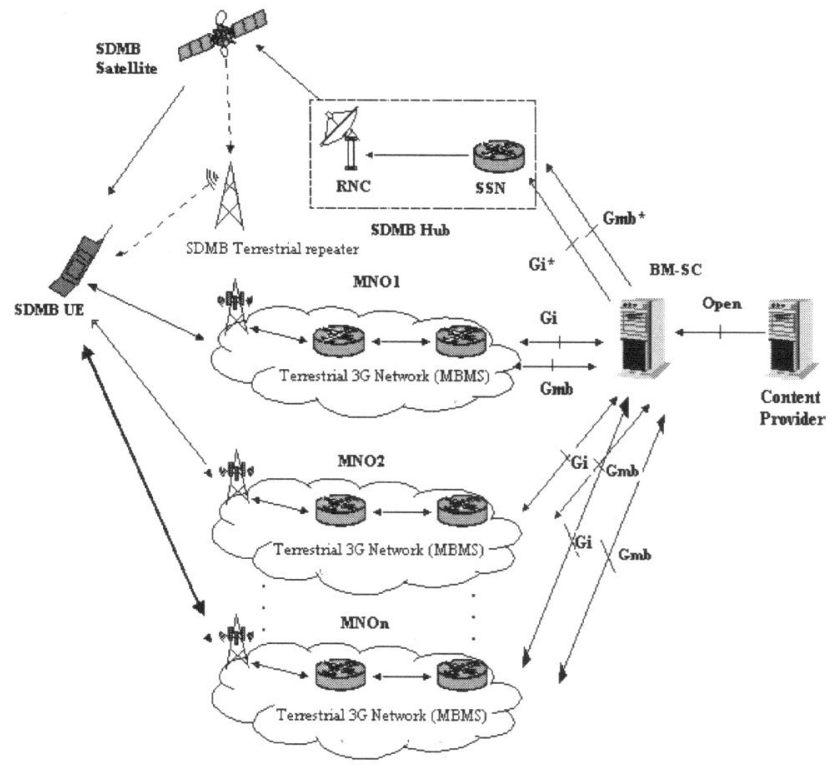

Figure 1. Integrated System architecture.

\section{LOCATION INFORMATION SIGNALLING}

Location information must be sent to the BM-SC by each users in a new specific message after the reception of the service announcement broadcast message.

In this way the BM-SC, knowing the amount of users and their location, can implement the following proposed strategy in order to minimize the transmission costs for both mobile and satellite network operators without to decrease the quality of service offered to the users.

Moreover the BM-SC could "periodically" estimate the number of connected users and their location deciding to switch the transmission bearer according to a new cost value.

The following time message scheme shows the communication between the UE, the Sat Hub and the BM$\mathrm{SC}$ highlighted the exchanged messages over different implicated interfaces.

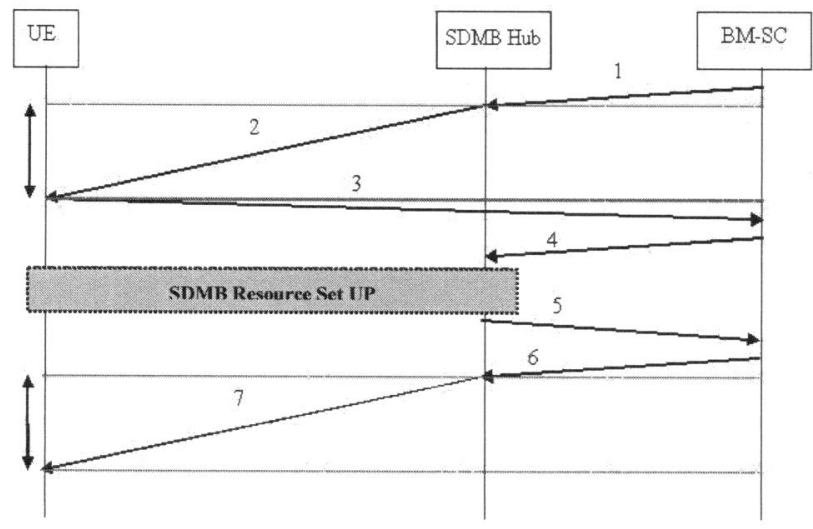

Figure 2. Session Start.
1. Service Announcement message sent over the Gi* interface between BM-SC and SDMB Hub

2. Service Announcement message sent through the SDMB to each UE in a broadcast way

3. New Answer UE message sent to BM-SC via return channel over Terrestrial UMTS network in order to inform the BM-SC about the availability to receive the service.

4. Session start request message sent over Gmb* interface

5. Session start response message sent over Gmb* interface

6. Data Transmission between BM-SC over Gi* interface

7. Data Transmission through the SDMB to each UE in a broadcast way

Due to the long round trip time delay between satellite hub and user terminal, it is better to sent the session start response message (5) after the SDMB Resource Set Up phase execution. In this way it is safely guaranteed that the necessary radio resources for the transfer of SDMB data to the interested UEs, will be established before the data transmission phase (6).

For the same reasons connected to the long round trip delay over the satellite link, this new time messages sequence must be adopted also in the session stop phase as shown in the next figure.

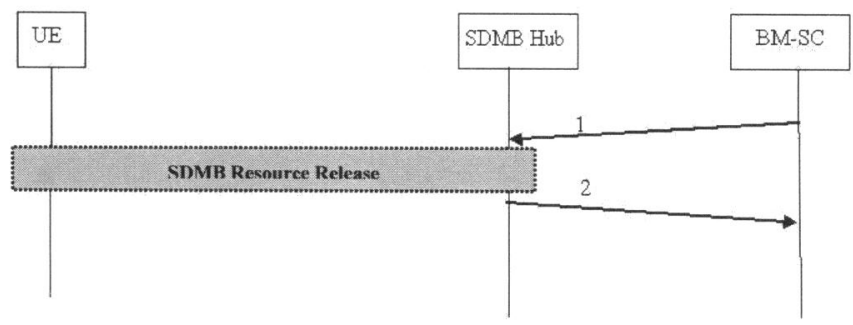

Figure 3. Session Stop.

1. Session stop request message sent over Gmb* interface

2. Session stop response message sent over Gmb* interface.

\section{COST MODEL FOR EACH MOBILE NETWORK OPERATOR}

Traditionally, cellular systems were designed to support voice services, so measures as production cost per minute, are usually employed when discussing the economies of such systems. We take the same approach when considering data services and derive the costs of supporting a certain user as being proportional with the usage time of the radio channel. In the followings, $\mathrm{Cu}$ is the average cost per second per user using the radio channel for a point to point unicast connection. Because the power consumption of radio transmitters in a cellular system does not represent a significant part of the costs, $\mathrm{Cu}$ mainly represents the cost per user as derived from the cost of necessary infrastructure deployment. This is because for a given user data rate the infrastructure cost grows linearly with the number of supported users, hence cost per user remains basically constant. For a given deployed infrastructure the cost of delivery the same multimedia item of size L (bits) to a number of $\mathrm{N}$ users through point to pint connection is:

Cost $=C u \cdot \sum_{j=1}^{N} \frac{L}{r_{j}}$

where $r_{j}$ is the data rate transmission of the $j^{\text {th }}$ connection. 
In this model, the cost for the channel usage per second is then directly proportional with the transmitted power: $\mathrm{Cb}=\alpha \mathrm{Pt}$. The cost of transmission for an item of length $\mathrm{L}$ (bits) with a data rate $\mathrm{Rb}$ for broadcast connection is:

$$
C_{\text {broad }}=C b \cdot \frac{L}{R_{b}}
$$

Intuitively, it should be possible to set a high rate for a subgroup of recipients experiencing good broadcasting link quality, while the rest will receive the item through terrestrial cellular unicast link.

Before introducing the optimization problem a few definitions and notations are necessary:

- Every multimedia item $i$ is characterized by a length $\mathrm{L}$ (bits) and is dedicated to a set of recipients $\mathrm{N}_{\mathrm{i}}$, of size $\mathrm{s}_{\mathrm{i}}$.

- The set of available data rates is $R_{b}$ for broadcasting and $R_{u}$ for unicasting.

- For each available broadcasting rate $R_{x} \in R_{b}$ and item $i$ there is a set of users $\mathrm{K}_{\mathrm{x}}$, of size $\mathrm{s}_{\mathrm{x}}$ that is able to receive the item with this rate if they met a certain signal-to-noise ratio required by $R_{x} \cdot K_{x}$ is a subset of $N_{i}$.

- The cost experienced by the operator when delivering the item $i$ is $\mathrm{C}_{\mathrm{MNO}}$. This cost is the sum of the cost for multicasting the item to the set $\mathrm{K}_{\mathrm{x}}$ using the broadcasting infrastructure and the cost of serving the set $\mathrm{N}_{\mathrm{i}}-\mathrm{K}_{\mathrm{x}}$ through point-to-point connections provided by the cellular infrastructure.

With these notations, the cost for each MNO can be written

$$
C_{M N O}=C b \cdot \frac{L}{R_{x}}+C u \sum_{j=1}^{s_{i}-s_{x}} \frac{L}{r_{j}}
$$

where $r_{j} \in R_{u}$ is the cellular data rate for each user $j$ in the set $s_{i}-s_{x}$. First term is the cost of broadcasting and the second one is the cost of using the cellular system. This expression is valid if $0<s_{x}<s_{i}$. For $s_{x}=s_{i}$ we have only broadcasting with the rate corresponding to the "worst" user. For $\mathrm{s}_{\mathrm{x}}=\mathbf{0}$ only the cellular system is employed.

The optimization problem is formulated as minimize $\mathrm{C}_{\mathrm{MNO}}$ by finding the optimum set $\mathrm{s}_{\mathrm{x}}$ and the corresponding rate $R_{x}$ such that all $N_{i}$ recipients eventually receive the item.

This problem has to be solved under different settings for number of item's recipients, certain values for $\mathrm{Cu}$ and $\mathrm{Cb}$, sets of available data rates, number of cells in the service area, cell radius, height of broadcasting tower, transmitted power, etc. Obviously under different settings the solution of the problem varies. Looking to the previous considerations, it is possible to note, that the proposed cost model is strictly connected to the service data rate. The service classes chosen for the SDMB network are Rich Media broadcast, Interactive TV broadcast, Data download and Group messages that support different application with different data rate; the table 1 is derived from [7] and shows the possible bit rate values for each application of the proposed SDMB network.

However, the previous considerations about the cost model are valid for a generic cellular network but in our studies we use a mobile 3G network based on WCDMA radio interface thus more attention must be addressed to the rate transmission values.

Wide-band code division multiple access (WCDMA) systems (e.g., ETSI WCDMA, cdma2000), which will be the major radio transmission technologies for IMT-2000, have intrinsic support for dynamic rate transmission. In a WCDMA system, with a fixed number of assigned codes, the uplink data transmission rate can be controlled on a frame-by-frame basis by using a variable spreading factor (VSF) method [8][9][10]; on the contrary the spreading factor and the number of codes in the downlink segment are fixed [11].

Nevertheless, using the WCDMA access scheme, the rate transmission value for each service class is not equal to the real data amount that must be send on the downlink segment because each connection needs to be spread on the entire transmission bandwidth (e.g. $5 \mathrm{MHz}$ ) using a suitable spreading factor value.

With the aim of conducting a first simple analysis, we impose the relation $R_{x}=r_{j}=R$ in the equation 3 considering the same data rate for unicast and broadcast connections and obtaining the following one:

$$
C_{M N O}=C b \cdot \frac{L}{R}+C u \sum_{j=l}^{s_{j}-s_{x}} \frac{L}{R_{j}}
$$

observing the two different contributions and knowing the ratio value between the broadcast and unicast costs, it is possible to derivate the following relation

$$
C_{M N O}=X \cdot C u \cdot \frac{L}{R}=C u \sum_{j=1}^{X} \frac{L}{R_{j}} \text { where } \frac{C b}{C u}=X
$$

We demonstrate that, for each MNO, the broadcast transmission cost is the same of the unicast transmission cost if the number of served users is equal to $X$, moreover if the number of active users in the MNO is equal to $\mathrm{Y}<\mathrm{X}$, it is better to use $\mathrm{Y}$ unicast connections rather than a single broadcast one.

Obviously the data rate is different for each SDMB application as shown in table I thus the generic proposed cost

\begin{tabular}{|c|c|c|c|c|}
\hline $\begin{array}{c}\text { SDMB } \\
\text { Services }\end{array}$ & $\begin{array}{l}\text { Service } \\
\text { Example }\end{array}$ & Media & $\begin{array}{c}\text { MBMS User } \\
\text { Service } \\
\text { Classification }\end{array}$ & $\begin{array}{c}\text { Application } \\
\text { Data rate }\end{array}$ \\
\hline $\begin{array}{l}\text { Rich media } \\
\text { Broadcast }\end{array}$ & $\begin{array}{c}\text { Audio } \\
\text { distribution } \\
\text { with low } \\
\text { quality video }\end{array}$ & $\begin{array}{l}\text { Stereo Audio, } \\
\text { Video (e.g. 3fps) }\end{array}$ & Streaming & Up to $128 \mathrm{kbps}$ \\
\hline $\begin{array}{l}\text { Rich media } \\
\text { Broadcast }\end{array}$ & $\begin{array}{c}\text { Video } \\
\text { distribution }\end{array}$ & $\begin{array}{c}\text { Video \& } \\
\text { supplementary } \\
\text { data (e.g. text, } \\
\text { still images) }\end{array}$ & Download & Up to $384 \mathrm{kbps}$ \\
\hline $\begin{array}{c}\text { Interactive } \\
\text { TV }\end{array}$ & $\begin{array}{c}\text { Video } \\
\text { streaming }\end{array}$ & $\begin{array}{c}\text { Video \& } \\
\text { supplementary } \\
\text { data (e.g. text, } \\
\text { still images) }\end{array}$ & Streaming & Up to $384 \mathrm{kbps}$ \\
\hline $\begin{array}{c}\text { Data } \\
\text { download }\end{array}$ & $\begin{array}{c}\text { General } \\
\text { Content } \\
\text { Distribution }\end{array}$ & $\begin{array}{l}\text { Video, Audio, } \\
\text { File Data (binary } \\
\text { data) }\end{array}$ & $\begin{array}{l}\text { Carousel, } \\
\text { download }\end{array}$ & Up to $384 \mathrm{kbps}$ \\
\hline $\begin{array}{c}\text { Data } \\
\text { download }\end{array}$ & $\begin{array}{c}\text { Audio } \\
\text { distribution } \\
\text { with low } \\
\text { quality video }\end{array}$ & $\begin{array}{l}\text { Stereo Audio, } \\
\text { Video (e.g. 3fps) }\end{array}$ & Streaming & Up to $128 \mathrm{kbps}$ \\
\hline $\begin{array}{c}\text { Data } \\
\text { Download }\end{array}$ & $\begin{array}{l}\text { Audio } \\
\text { download }\end{array}$ & Stereo Audio & Download & Up to $48 \mathrm{kbps}$ \\
\hline $\begin{array}{c}\text { Group } \\
\text { Messages }\end{array}$ & $\begin{array}{l}\text { Reliable text } \\
\text { distribution (eg. } \\
\text { Local news) }\end{array}$ & Text & Download & Up to $10 \mathrm{kbps}$ \\
\hline $\begin{array}{c}\text { Group } \\
\text { Messages }\end{array}$ & $\begin{array}{c}\text { Text } \\
\text { distribution } \\
\text { with still } \\
\text { images and/or } \\
\text { low quality } \\
\text { video }\end{array}$ & $\begin{array}{l}\text { Text, Still } \\
\text { images, Video } \\
\text { (e.g. 3fps) }\end{array}$ & $\begin{array}{l}\text { Carousel, } \\
\text { download }\end{array}$ & Up to $32 \mathrm{kbps}$ \\
\hline
\end{tabular}
model can be used for estimating different cost values.

TABLE I

SDMB APPLICATIONS AND PARAMETERS.

\section{DECISION STRAT EGY AND RESOURCES ALLOCATION FOR THE BM-SC (AGGREGATOR CENTRIC)}

In our studies we used a network architecture role model based on Aggregator Centric [1]; key features of this architecture are that the MNO still owns the SDMB user but the MNO is not responsible for the delivery of SDMB services to the users. This role is performed by a separate entity (the Aggregator) which hosts same functional aspects 
of the BM-SC including content aggregation, conditional access and reliable transport.

This role model may have significant advantages in enabling the same content to be sold to many operators, thus making more efficient use of satellite bandwidth.

For each SDMB service or application we need to evaluate the cost function of each $\mathrm{MNO}$, it is also necessary to evaluate the Utilization Factor or Congestion Factor for each MNO [12] in order to decide the best path over a satellite or terrestrial bearer for an SDMB service.

The cost function for each MNO is already shown in (5) whilst the cost function for the broadcast transmission over the satellite bearer is:

$$
C_{\text {broad }-S A T}=C s \cdot \frac{L}{R_{B S}}
$$

where $\mathrm{C}_{\mathrm{S}}$ is the cost for the satellite channel usage per second that is bigger than $\mathrm{Cb}$ and $\mathrm{Cu}$ and represent an other study parameter [13].

Two different cases have been investigated:

1. No Mobile Network is congested

2. At least one Mobile Network is congested

It is necessary to fix an utilization threshold value for each MNO in order to evaluate the congestion state and to operate the right choice.

In the first case, because no mobile network is congested, it is possible to try to allocate traffic resources over the terrestrial bearer evaluating the cost functions in the following way:

$$
\text { if }\left(\sum_{i=1}^{\# M N O} C_{M N O i}<C_{\text {broad-SAT }}\right)
$$

then Resources Allocation over T-UMTS MNOs

Else if $\mathrm{d}$ (expected delivery delay $)<$ delaySatRTT

Resources Allocation over T-UMTS MNOs Else

Resources Allocation over Satellite bearer

In order to increase the QoS offered to the users reducing the delivery delay for each application, we always prefer to use the T-UMTS network even if it is not very profitable for the Mobile Network Operator. However the expected delivery delay for the chosen applications is always greater than the satellite round trip time delay because this broadcast system is designed for not strictly real time services like rich media broadcast, interactive TV, data download and group messages that can support an IP delay variation in a range between $400 \mathrm{~ms}$ up to $1 \mathrm{sec}$.

In the second case, if one of the mobile networks is congested, we choose to send data using only the satellite broadcast bearer for all the users of all mobile networks. Obviously this is one of the possible choice, we don not say that it is the best choice, it could be also possible to send data using the congested mobile network infrastructure decreasing the QoS offered to the users and saving the satellite link bandwidth.

\section{Simulation Results}

In our simulation campaign we perform a snapshot analysis (steady state) with maximum fixed number of MNOs and users in each of them. In each snapshot one multimedia SDMB service is delivered to the recipients in the service area.

The service consists of delivery of a multimedia item $i$ that is characterized by its size in bits $\mathrm{L} i$ and number of recipients ni. Each mobile user has a terminal that is always on and able to connect simultaneously to both terrestrialUMTS (unicast-broadcast) and Satellite (broadcast) systems. We only consider the downlink segment. The main simulation parameters are summarized in table II.

TABLE II

SiMULATION PARAMETERS

\begin{tabular}{cc}
\hline $\mathrm{Cb} / \mathrm{Cu}$ & $30-50-80-120-150$ \\
$\mathrm{Cs}$ & 220 \\
\# of MNOs & 5 \\
Multimedia Service Size $\mathrm{Li}$ & $3 \mathrm{Mbits}$ \\
Multimedia Service Data Rate & $384 \mathrm{kbps}$ \\
Percentage of users asking for & $10 \%-20 \%-30 \%-40 \%-50 \%$ \\
SDMB Services & Exponential Distributed \\
Maximum number of users for each & 5000 \\
MNO & $80 \%$ \\
Congestion MNO threshold
\end{tabular}

Figures 3 and 4 show the system behavior when no mobile network is congested. In particular figure 3 highlights in which conditions, in term of number of users asking for a SDMB service, is better to use terrestrial UMTS broadcast resources allocation rather than the unicast one. The curves are obtained varying the $\mathrm{Cb} / \mathrm{Cu}$ parameter that is a distinctive MNO parameter.

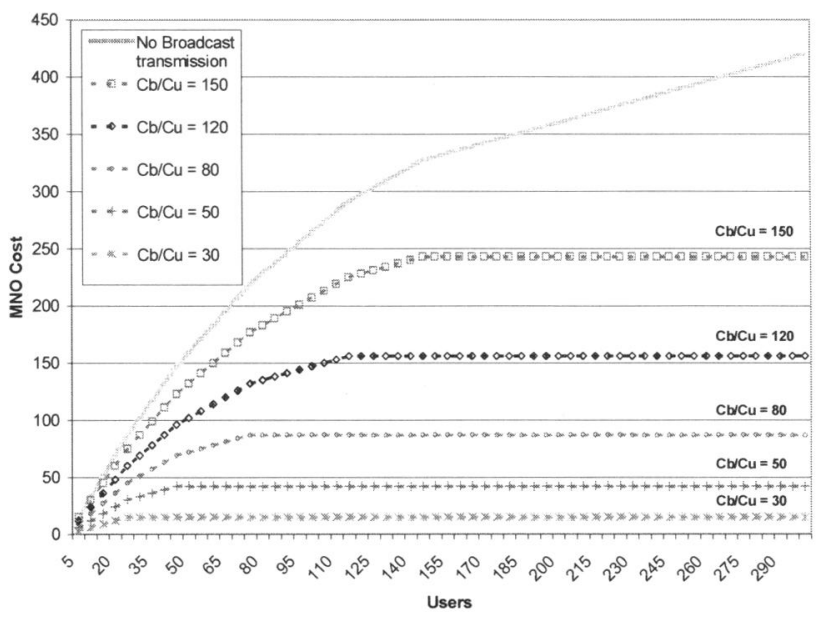

Figure 3. Costs for each MNO.

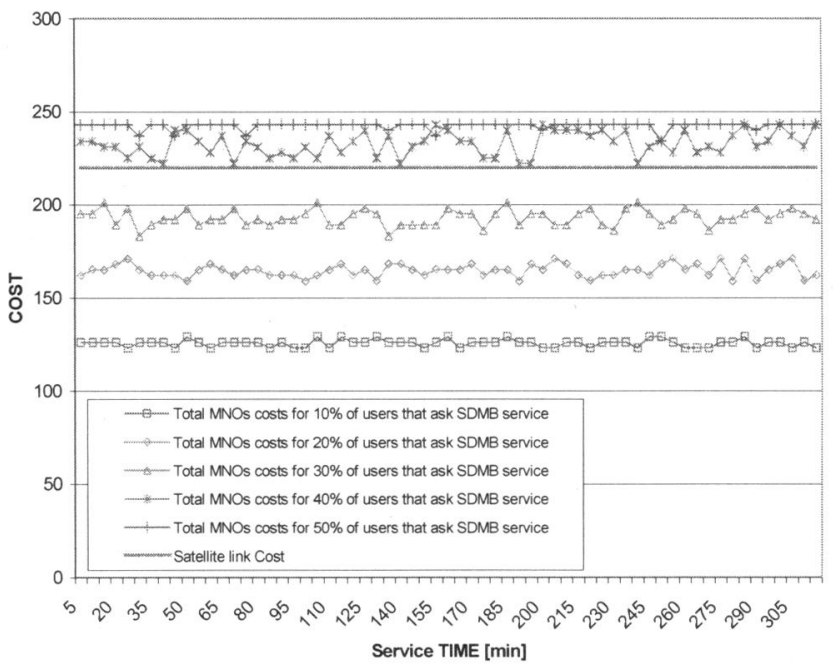

Figure 4. Total MNOs cost varying the percentage of users asking for SDMB service. 
Figure 4 shows the total MNOs cost varying the percentage of users belonging to each $\mathrm{MNO}$ and asking for the SDMB service. Obviously the BM-SC has to manage all the five mobile networks that have different $\mathrm{Cb} / \mathrm{Cu}$ values. It is possible to observe that, using the simulation parameter in table II, if the percentage of users asking for the SDMB services is equal or greater than $40 \%$, it is more convenient to send data to all MNOs through the satellite broadcast link. Clearly the cost model analysis is always valid but the obtained results are strictly linked to the input parameters that can be changed according to the real constrains of each mobile or satellite network operator.

Figure 5 shows the congestion level for each MNO versus the SDMB service time is, obviously the congestion trend is really unpredictable because it is function of many factors like number of users, mobility and day time. Moreover the figure highlights the maximum congestion level that represents, in each time, the MNO that is more congested.

This parameter is used by the BM-SC in order to make the efficient choice for sending data over the terrestrial or satellite broadcast link as already explained; if the maximum congestion value is greater than the fixed congestion threshold value, then the BM-SC chooses to send data using the broadcast satellite link with the aim of saving the QoS level offered to the users in the congested mobile network.

Obviously this is only one possible approach; it should be possible to send data using the terrestrial UMTS network even if it is congested, in this case a deep and accurate study on the QoS degradation for the users belonging to the congested mobile network, should be necessary.

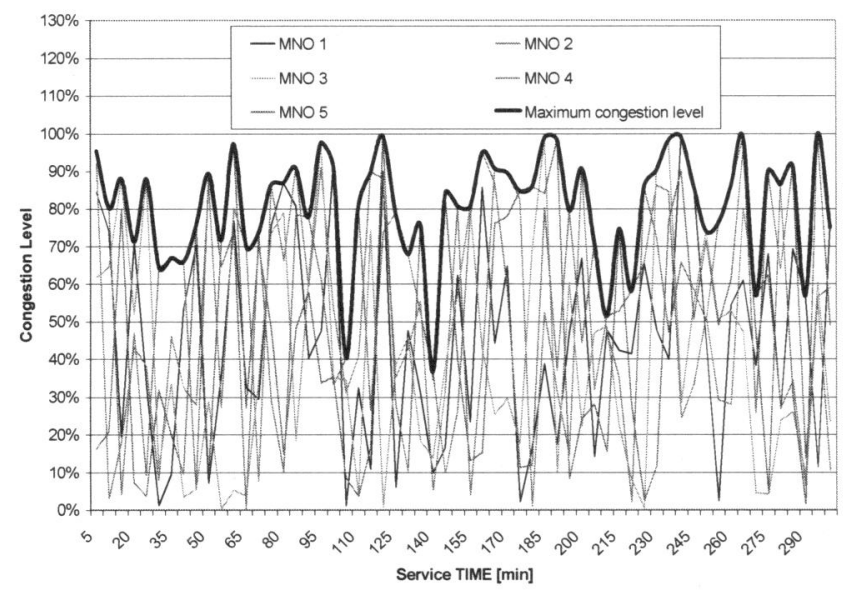

Figure 5. Congestion level for each MNO.

Figure 6 is a snapshot of the BM-SC cost variation when the choice is conducted with and without the information about the congestion value of the MNOs.

When no congestion has been found, the transmission cost is the sum of the costs of each mobile network whilst when at least one mobile network is congested, the transmission cost is a fixed cost due to the broadcast satellite link.

\section{CONCLUSION AND DISCUSSION}

This paper proposes a simple and efficient cost model for choosing the more suitable transmission bearer (satellite or terrestrial) in an integrated satellite-terrestrial UMTS architecture.
The conducted studies and the obtained results show how it is possible to evaluate the best unicast or broadcast allocation for each mobile network depending by the total cost function and varying the $\mathrm{Cb} / \mathrm{Cu}$ parameter; moreover it is possible to choose the minimum cost bearer according to the percentage of users that ask for a specific offered SDMB service.

The gains obtained by inter-working terrestrial and satellite broadcast access should then be investigated in conjunction with the available information about radio links; unfortunately, this information might be incomplete, delayed or not available at all.

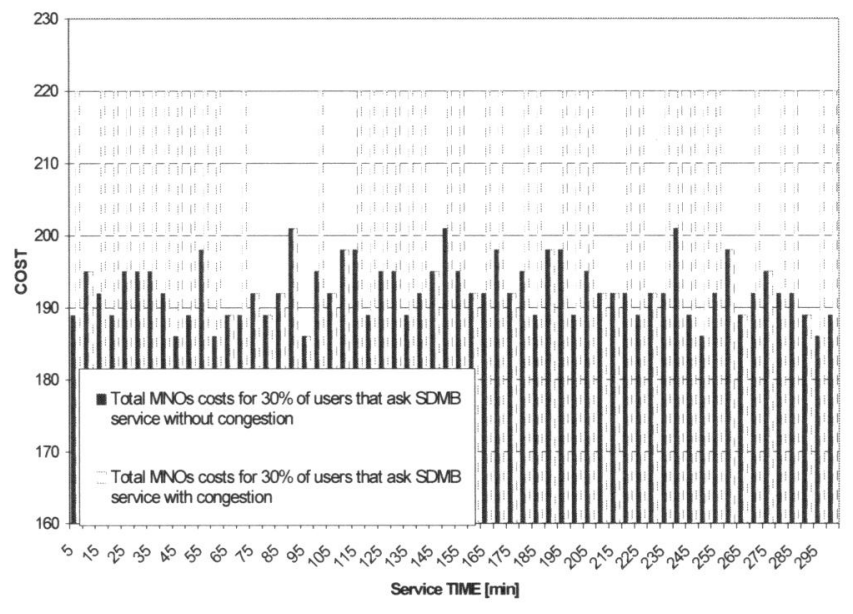

Figure 6. Congestion level for each MNO.

\section{REFERENCES}

[1] Mobile Applications \& sErvices based on Satellite \& Terrestrial inteRwOrking project; http://ist-maestro.dyndns.org/MAESTRO/

[2] Broadband services for everyone over fixed wireless access networks: BROADWAN project; http://www.telenor.no/broadwan/

[3] Stratospheric Broadband CAPANINA project; http://www.capanina.org/

[4] N. Chuberre, G. E. Corazza, MG Francon, C. Nussli, C. Selier, A. Vanelli-Coralli, P. Vincent, "Satellite Digital Multimedia Broadcasting for $3 G$ and beyond $3 G$ systems" $13^{\text {th }}$ IST Mobile \& Wireless Communications Summit 2004, Lyon, France, June 2004.

[5] K. Narenhiran et al., "S-DMB System Architecture and the MODIS DEMO" IST Mobile and Wireless Telecommunications Summit, Aveiro, Portugal, June 2003.

[6] V.Kueh, M.Karaliopoulos, B.G.Evans, P.Vincent, G.Chengeleroyen J.Malsch, N. Koutsokeras, E. Angelou, I.Andrikopoulos "Satellite Digital Multimedia Broadcast (SDMB) Access Layer Definition", $6^{\text {th }}$ EMPS \& ASMS, Noordwijk/NL, September 2004, ESA/ESTEC.

[7] 3GPP TS 22.246 V6.2.0 (2004-09) Technical SpecificationGroup Services and System Aspects; "Multimedia Broadcast/Multicast Service (MBMS) user services", Stage 1 (Release 6).

[8] E. Dahlman et al., "UMTS/IMT-2000 based on wideband CDMA" IEEE Commun. Mag., vol. 36, pp. 70 80, Sept. 1998.

[9] E. Dahlman and K. Jamal, "Wide-band services in a DS-CDMA based FPLMTS system”, Proc. IEEE VTC '96, pp. 16561660.

[10] S. Nanda, K. Balachandran, and S. Kumar, "Adaptation techniques in wireless packet data services", IEEE Commun. Mag., Jan. 2000.

[11] D. In Kim, E. Hossain, V.K. Bhargava, "Dynamic rate adaptation and integrated rate and error control in cellular WCDMA networks" Wireless Communications, IEEE Transactions on, Volume 3, Issue 1, Jan. 2004, pp. $35-49$.

[12] A. Bria, "Multi-radio Resource Management in Hybrid CellularBroadcasting Networks", PCC Personal Computing and Communication Workshop, Stockholm, November 2003.

[13] B. Xu, O. Wolfson, S. Chamberlain, N. Rishe, "Cost based data dissemination in satellite networks" Mobile Networks and Applications - Kluwer Academic Publishers, Volume 7, pp.49-66, January 2002. 